\title{
La ética y la equidad como ejes de la cooperación en salud global
}

\author{
GIORGIO SOLIMANO $^{(1)}$ y LEONEL VALDIVIA $^{(1)}$
}

El desarrollo y la cooperación en salud global sólo son factibles desde una visión compartida de la ética y de la equidad. Esto es especialmente válido para la colaboración sur-sur, ya que en la colaboración norte-sur hemos encontrado diferencias con la ética que predomina en el norte la que se basa, principalmente, en el ejercicio del individualismo y una preocupación mayor por la eficiencia (especialmente de costos), que por la búsqueda de la equidad y la solidaridad en el campo de la salud. Por otra parte, es justo reconocer que en los últimos años, académicos de Canadá y Estados Unidos de Norteamérica han evolucionado hacia una visión ética de la salud pública focalizada en la búsqueda de la equidad y la "justicia social" como lo describe Dan. E. Beauchamp en su trabajo fundacional "Public Health and Social Justice". Esta posición supera el tradicional énfasis en las libertades individuales que predominaba en los colegas del norte.

En este contexto, es posible reconocer huellas del camino recorrido por nuestra Escuela, implícitas en su historia, que nos ha llevado en los últimos años a sentar las bases de un Programa de Salud Global. A continuación, identificamos y reseñamos brevemente cuatro períodos en el desarrollo de la Escuela que se relacionan con este campo.

\section{3-1973}

En 1943 se funda la Escuela de Salud Pública con apoyo de la Fundación Rockefeller (cooperación norte-sur). En la década de los
40 e inicio de los 50 se gesta la creación del Servicio Nacional de Salud, proceso al que la Escuela de Salud Pública contribuye con la formación de salubristas chilenos y también latinoamericanos (cooperación sur-sur). En la década de los 60, se implementan los programas de "control de la fertilidad" con financiamiento internacional (cooperación norte-sur condicionada). Durante la Presidencia del Dr. Salvador Allende -1970-1973-, se debilita la colaboración norte-sur pero aumenta la solidaridad de grupos progresistas tanto en el norte como en el sur.

\section{3-1990}

Durante la dictadura militar se observa un debilitamiento de la salud pública chilena y de la Escuela, asociado al aislamiento internacional del régimen. Numerosos salubristas parten al exilio y se desarrolla una fuerte solidaridad internacional. Igualmente se fortalece la colaboración norte-sur y sur-sur sobre ética compartida, destacando la defensa de los derechos humanos y la solidaridad. Por otra parte, Chile ingresa tempranamente a la globalización económica neoliberal impuesta y vigilada con mano militar. En este período, aun cuando existe evidente crecimiento económico, la inequidad social aumenta y predomina la mercantilización de la salud.

\section{0-2003}

El año 1990 marca el inicio de la transición a la democracia incluyendo el retorno de nu-

(1) Escuela de Salud Pública. Facultad de Medicina. Universidad de Chile. Independencia 939. Independencia. Santiago.Chile.gsolimano@med.uchile.cl 
merosos salubristas exiliados. En el ámbito académico, en 1999 se inicia un período de recuperación y modernización de la Escuela sustentado en planes estratégicos de desarrollo formulados en forma participativa. A partir de ese momento se generan vínculos internacionales y proyectos colaborativos, destacando el ejercicio de la Presidencia de Alaesp durante el período 2000-2006 (un ejemplo de cooperación sur-sur). Igualmente, a partir de 1999 se prioriza el fortalecimiento y renovación del cuerpo académico, la creación del Programa de Doctorado en Salud Pública y de la Escuela Internacional de Verano en alianza con instituciones académicas extranjeras de excelencia y énfasis en los temas de ética y equidad en salud (cooperación norte-sur cualitativamente diferente).

\section{3 a la fecha}

El año 2003 se crea la Coordinación de Relaciones Internacionales en la Escuela como un esfuerzo orientado a consolidar la cooperación con centros de excelencia en la Región de las Américas y de Europa. El 2005 se lanza en Chile la Comisión sobre Determinantes Sociales de OMS, y el año 2008 la Escuela ingresa al Trade \& Health Forum de la Asociación Americana de Salud Pública -APHA-, iniciándose la dictación de cursos sobre los efectos de la globalización económica en la salud pública. En abril de 2009, se comienza formalmente a trabajar en la creación del Programa de Salud Global, el que es oficialmente ratificado por las instancias directivas de la Escuela en enero de 2011.

Esta breve reseña de nuestro caminar hacia la salud global muestra que la lucha por mayor equidad en salud y la salvaguardia de una ética de la salud pública, han estado presentes en el desarrollo de la Escuela, en el entendido que en Chile el quehacer relacionado con la salud global se ha desenvuelto en un contexto complejo y desafiante.

Desde la década de los 70, Chile se abrió al proceso de globalización económica y el libre mercado con fiel aplicación de todos los prin- cipios de la doctrina del neoliberalismo. Este período se caracterizó por un debilitamiento del Estado en su rol regulador y más aún en su rol de protector social. En este contexto, la salud al igual que la educación y la seguridad social pasaron a ser "mercancías" transables en el mercado y sujetas a sus reglas, con el consecuente agravamiento de la inequidad social en general y de la salud en particular.

Aunque a partir de 1990 los gobiernos democráticos han realizado importantes cambios en pro de la protección social, el sello de ese sistema está aún presente en la sociedad chilena. El tema "acceso a medicamentos" nos brinda un buen ejemplo de inequidad en salud pública con claros determinantes globales, como son las políticas y normas de protección de patentes y las utilidades de las grandes empresas farmacéuticas transnacionales. Para muestra un botón, el programa de VIH/Sida de Chile asigna la mayor parte de sus recursos a la adquisición y distribución de la terapia retroviral y a pagar al personal médico y paramédico necesario para su administración y monitoreo. Desafortunadamente, los programas de prevención y consejería no se benefician en igual medida. En gran parte, esto se debe a los elevados precios de los medicamentos patentados y a la falta de acceso a genéricos, debido a las limitaciones impuestas por el sistema de patentes al que Chile está adscrito. Claramente, este es un problema de carácter global y debe enfrentarse de igual manera. Pero no sólo eso, debe enfrentarse también a partir de una consideración ética vinculada al derecho de las personas y a la búsqueda de equidad en el acceso a los medicamentos y al mejoramiento de la salud en general.

Otro ejemplo ilustrativo dice relación con la "investigación en salud global", que para nosotros no es otra cosa que investigación en salud pública (como la hemos hecho desde hace muchas décadas), pero con una mirada transnacional, es decir identificando los determinantes y las soluciones a problemas de salud en el contexto global. Históricamente las escuelas e institutos de salud pública 
latinoamericanos han actuado de anfitriones de proyectos de investigación generados en instituciones del norte, sin que esto signifique desconocer el valioso aporte de colegas de Europa y Norteamérica a nuestro progreso científico. Pero también debemos someter dichos vínculos a un serio escrutinio desde una perspectiva ética y de equidad. Al respecto, Saul Franco, colega colombiano ("Ética de la investigación en salud pública") respecto al rol que cumplimos o podemos cumplir en investigación, plantea dos tajantes preguntas: "¿somos sujetos activos o potenciales pasivos implementadores de algunas técnicas en serie? y ¿somos sujetos de los usos saludables de los avances logrados, o sujetos de experimentación menos costosos que ciertos ratones de laboratorio primer mundista?". Sin duda, estas interrogantes nos obligan a no permitir que la colaboración sur-sur genere cuestionamientos de esta naturaleza, sino que en ella predominen sólidos principios compartidos de ética y equidad.

Estoy cierto que situaciones como éstas, con características propias y diferentes magnitudes existen en diferentes medios, generando problemas que trascienden fronteras y que es nuestra responsabilidad abordar en el contexto de la salud global hemisférica

La ética, como se dijo al inicio, constituye el segundo eje fundamental para el desarrollo de la salud global, disciplina que inicialmente se desarrolló casi exclusivamente en el dominio de la medicina clínica y experimental, pero que con el correr de los años se ha ampliado generando la ética de la salud pública, la que progresivamente ha adquirido individualidad y un sólido basamento conceptual en Latinoamérica. A ello contribuyen colegas de Argentina, Brasil, Chile, Colombia y México entre otros, que en los últimos años han fortalecido el andamiaje de la bioética latinoamericana con características que la diferencian claramente de la bioética norteamericana.

Sobre los alcances de la ética latinoamericana, el reciente artículo de Jennifer Bulcock: "The many beginnigs of bioethics: A comparison of American and Ibero-Ameri- can bioethics and the possibility of a Global Bioethics", plantea en forma fundamentada cuatro características que diferencian la bioética latinoamericana de la americana: 1 . La primera constituye una ética comunitaria versus una ética individualista (centrada en la autonomía y los derechos y responsabilidades individuales). 2. En el caso de la bioética latinoamericana se trata de un colectivo encabezado y guiado por médicos y teólogos versus un colectivo motivado por filósofos y académicos. 3. La latinoamericana es una bioética concebida como un movimiento social versus una disciplina académica institucionalizada con aplicaciones prácticas. 4. El contenido de la bioética latinoamericana está directamente relacionado con la historia social, económica, cultural, política y religiosa y el tipo de desarrollo de una determinada región o país.

Como se ve las diferencias no son menores y tampoco excluyentes ya que nuestros países, dependiendo de la problemática que se trate, requieren de ambas. Sin embargo, la perspectiva latinoamericana satisface y se corresponde mejor con el desarrollo de la salud global y el fortalecimiento de la cooperación sur-sur, que privilegia el logro de la equidad en salud, ámbito en el cual es necesario ampliar y fortalecer la colaboración.

Con el fin de avanzar en este sentido, entre muchas iniciativas posibles, las tres que se enuncian aparecen como factibles en un corto plazo:

1. Promover un proceso de reflexión e investigación en salud global a partir del rescate histórico del pensamiento latinoamericano sobre la ética de la salud pública y la equidad, así como de nuevos análisis sobre nuestra realidad en constante evolución para conformar un corpus latino americano de conocimiento en salud global.

2. Establecer una forma de organización latinoamericana en el campo de la salud global, que permita la colaboración regular y sustentable entre instituciones académicas comprometidas con este tema en nuestra Región. 
3. Promover el desarrollo de un modelo curricular para la enseñanza de la salud global, basado en la ética y la equidad en salud desde una perspectiva latinoamericana haciendo uso de las metodologías que la moderna tecnología de la información nos ofrece.

Este artículo fue adaptado de la presentación al Primer Congreso Latinoamericano de Salud Global, Cuernavaca, México, abril 2010.

Usted puede comentar éste y otros artículos publicados en la Revista Chilena de Salud Pública, enviando un correo electrónico a revistasp@med.uchile.cl 